\section{Design and performance of a large lumen glaucoma drainage device}

\begin{abstract}
Purpose We report the in vivo testing of a large-lumen glaucoma drainage (LL-GDD) device equipped with a flow regulator. The device's membrane can be non-invasively opened with laser in the postoperative period to adjust aqueous flow and intraocular pressure. Methods The initial LL-GDD prototypes were constructed using $22 \mathrm{G}$ silicone angiocatheters cut down to size. A $10 \mathrm{~nm}$ PVDF membrane was then affixed to the end using cyanoacrylate. The LL-GDD was tested first in a model eye equipped with ports for infusion and pressure measurement and in New Zealand rabbits.

Results New Zealand white satin cross rabbits were used, two eyes receiving the LL-GDD and the two fellow eyes serving as the control group with no intervention performed. After the procedure, the IOP in the LL-GGD surgical group dropped an average of $5.5 \mathrm{~mm} \mathrm{Hg}$ $(P=0.001)$, which was maintained until the membrane laser procedure at week 5 resulting in an average IOP reduction of $1.8 \mathrm{~mm} \mathrm{Hg}$. At week 7, the average IOP in the surgical group was $11 \mathrm{~mm} \mathrm{Hg}$ compared with $18 \mathrm{~mm} \mathrm{Hg}$ in the control group $(P<0.001)$. A second laser procedure was done to completely open the membrane face, which resulted in an immediate drop in the average IOP of the surgical group by another $2.7 \mathrm{~mm} \mathrm{Hg}$, which was maintained until the study termination at day 55 . Conclusions The large-lumen glaucoma drainage device demonstrated an ability both to prevent immediate postoperative hypotony and to allow progressively lower IOP on demand in this proof-of-concept study. Eye (2017) 31, 152-156; doi:10.1038/eye.2016.182; published online 9 September 2016
\end{abstract}

Fax: +1 7208485014

E-mail: sergio.groman-

lupa@ucdenver.edu

Received: 29 April 2015 Accepted in revised form: 19 May 2016 Published online: 9 September 2016
JL Olson and S Groman-Lupa

nerve. In the United States, glaucoma represents the second leading cause of legal blindness, despite current treatment and management protocols. ${ }^{1}$ The primary goal in the treatment of glaucoma is to prevent or delay the loss of visual function caused by damage to the optic nerve. Although several risk factors for developing glaucoma have been identified, therapeutic approaches are currently limited to reducing intraocular pressure (IOP). ${ }^{2}$ Traditionally, the initial standard therapy is medical; however, when patients have new or progressive field loss whether from poor compliance, inadequate IOP reduction, or simply uncontrolled disease, laser or incisional surgeries are indicated. ${ }^{3}$

Trabeculectomy is the most frequently performed filtering operation, and remains one of the most effective operation. However, it can be complicated by choroidal detachment or endophthalmitis, even years after surgery. ${ }^{4}$ Other options such as non-penetrating filtration surgery (deep sclerectomy and canaloplasty) have the advantage of not entering the anterior chamber but involve a steep learning curve of the technique and frequently require adjunctive medications or laser, while producing a less pronounced effect on IOP than trabeculectomy. ${ }^{5}$ In addition, procedures and devices designed to shunt fluid from the anterior chamber directly into Schlemm's canal are available, but produce modest benefits as well. ${ }^{6}$

Glaucoma drainage devices (GDD) have shown an advantage in maintaining IOP control compared with trabeculectomy for patients with uncontrolled IOP after previous incisional surgeries. ${ }^{7}$ This has resulted in an increased interest in the use of GDD for the management of glaucoma and is the option of choice for many types of glaucoma such as neovascular, uveitic, iridocorneal endothelial syndrome, glaucoma related to penetrating keratoplasty, keratoprosthesis, or following retinal detachment repair.

The most common early complications of tube shunt implantation are hypotony and associated problems. 8,9 The Glaucoma Drainage Device Regulator (GDDR) implant described here was
Glaucoma is characterized by a disruption of the structural and functional integrity of the optic 
designed to overcome these hurdles. It allows the surgeon to control the rate of flow through the device noninvasively in the postoperative period, allowing customized treatment for patients (Figure 1).

Previously, we have reported on a $23-\mathrm{G}$ flow regulator, which can be used on conventional drainage tubes. ${ }^{10}$ Current commercially available shunts typically use a silicone tube with an outer diameter of $0.64 \mathrm{~mm}$ (23 G) and an inner diameter of $0.34 \mathrm{~mm}$ ( $30 \mathrm{G})$. Herein, we describe a second generation device with an increased lumen size: the large lumen glaucoma drainage device (LL-GDD), which has an outer diameter of $0.72 \mathrm{~mm}$ (22 G) and an internal diameter of $0.5 \mathrm{~mm}$. This represents an increase in the outer diameter of $13 \%(0.08 \mathrm{~mm})$ and an increase in the inner diameter of $47 \%(0.16 \mathrm{~mm})$, which translates into a quadrupling of flow as described by Poiseuille's law whereby there is an exponential increase in flow with relation to the tube radius described by the equation: ${ }^{11}$

$Q=\frac{\pi \operatorname{Pr}^{4}}{8 \eta l}$

With conventional implant hardware designs, this enlarged lumen device could not be safely placed in an eye, as the high rate of uncontrolled flow in the immediate postoperative period would lead to profound hypotony. However, using the glaucoma drainage device regulator (GDDR) technology, this additional flow can be controlled and held in reserve. That is, postoperatively the flow is restricted by the device's membrane, which covers the lumen of the drainage device. As clinical conditions demand, the membrane can be non-invasively opened with laser. We have demonstrated in previous studies that the membrane reduces, but does not totally restrict flow when completely intact. ${ }^{10}$ This is advantageous as it allows immediate IOP control, as well as keeping aqueous flowing through the device to prevent blockage or failure of the GDD and to prevent infection.

The goals of this proof-of-concept study were to: (1) demonstrate surgical implantation; (2) prevent immediate postoperative hypotony; (3) increase flow on-demand post implantation; (4) compare flow rates to conventional drainage devices.

\section{Materials and methods}

The initial LL-GDD prototypes were constructed using $22 \mathrm{G}$ silicone angiocatheters cut down to size (Figure 2). A $10 \mathrm{~nm}$ PVDF membrane was then affixed to the end using cyanoacrylate. PVDF was chosen given its long track record of biocompatibility and previous use in intraocular lens designs. Further, the membrane's thickness allows it to be easily ruptured using either thermal or photodisruptive lasers. Using a standard Baerveldt (Abbott Laboratories, Abbott, IL, USA) drainage device, the standard $23 \mathrm{G}$ tube was removed and the $22 \mathrm{G}$ tube affixed to the reservoir plate.

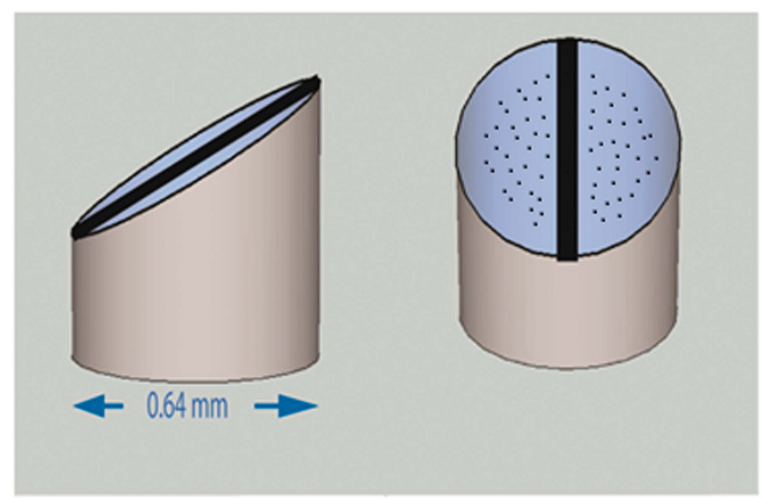

Figure 1 The Glaucoma Drainage Device Regulator. The device fits over the distal end of a glaucoma tube when placed in the anterior chamber. Postoperatively, the membrane face is punctured with laser or a needle to increase flow through the tube.

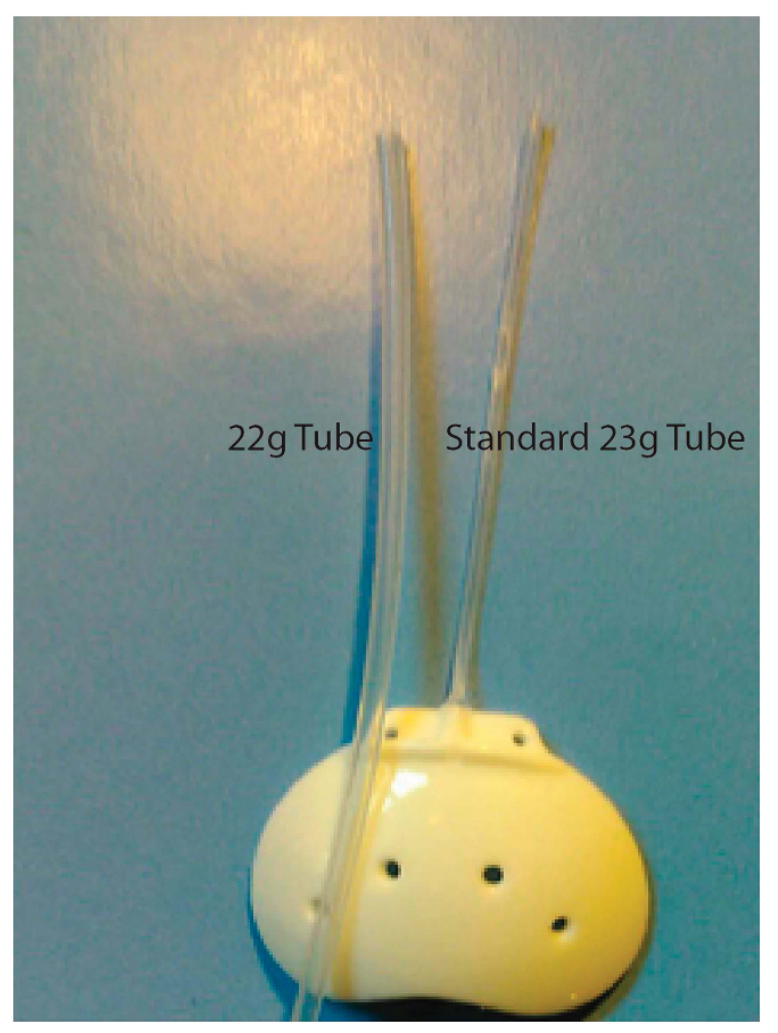

Figure 2 A 22 G silicone tube (left) compared with the standard $23 \mathrm{G}$ (right). The $47 \%$ increase in the internal diameter results in a 4.7-fold increase in potential flow through the tube. 


\section{In vitro testing}

The LL-GDD was tested first in a model eye equipped with ports for infusion and pressure measurement. Balanced saline solution was hung at the appropriate height to maintain a constant pressure of $25 \mathrm{~mm} \mathrm{Hg}$, which was monitored during the testing using an industrial grade differential pressure manometer (HD750, Extech Insturments, Nashua, NH, USA). The LL-GDD prototype was placed into the system and the amount of fluid, which passed through the tube was measured for $30 \mathrm{~s}$. The membrane was placed initially with no laser perforations, then with enough laser to progressively open $1 / 6$ of the membrane until $100 \%$ of the membrane was opened. An Nd:YAG laser (YC-1600, Nidek, INc, Fremont, CA, USA) was used to rupture the PVDF membrane with the following parameters: $4.3 \mathrm{~mJ}$, single pulse. Further, a conventional $23 \mathrm{G}$ tube was tested with no regulator in place as a control. Three measurements were done for each configuration, and the results averaged.

\section{In vivo testing}

The study was reviewed and approved by the University of Colorado Institutional Animal Care and Use Committee. All procedures were performed according to the statement for the use of animals in ophthalmic and visual research from the Association of Research of Vision and Ophthalmology. The study was conducted at the University of Colorado facilities and was funded by the Colorado Bioscience Discovery Grant.

New Zealand white satin cross rabbits were used, two eyes receiving the LL-GDD and the two fellow eyes serving as the control group with no intervention performed. For all surgical cases, the conjunctiva was opened at the limbus for three clock hours superonasally and the underlying sclera exposed. To accommodate the decreased size of the rabbit's globe, all of the reservoir plates were cut down $2 \mathrm{~mm}$ on each side using a template to ensure consistency. The reservoir plate was affixed to the globe using 8-0 nylon suture. A $22 \mathrm{G}$ needle was used to create a tunnel through the sclera and enter the anterior chamber just anterior to the iris. This tunnel was widened slightly in the large lumen device group to accommodate the larger tube. The tubes were then placed in the anterior chamber and the conjunctiva repositioned with vicryl suture (Figure 3). At postoperative weeks 5 and 7, the membrane on the $22 \mathrm{G}$ device was ruptured with argon laser.

In all animals the right eye underwent surgery and the left eye served as control. All eyes undergoing surgery received topical antibiotic drops for 7 days and topical steroid drops for 2 weeks. Baseline IOP and anterior

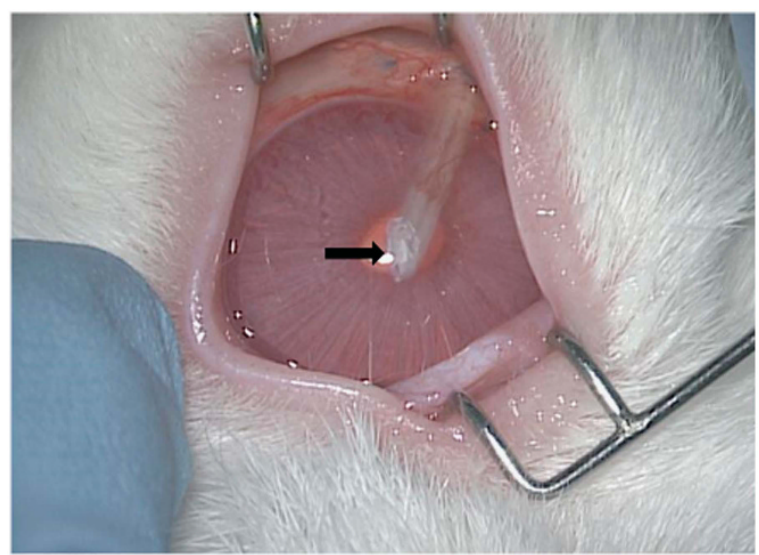

Figure 3 LL-GDD implanted in a rabbit eye with the GDDR flow restrictor membrane (arrow) visible in the anterior chamber.

segment photos were taken from all eyes, and IOP taken immediately before and after every procedure, as well as twice a week for 8 weeks of the study. A hand-held veterinary model tonometer (Tono-Pen Vet, Reichert Technologies, Depew, NY, USA) was used for this purpose. The drainage devices were left in place for the duration and the animals examined daily for the first week and then weekly thereafter. The Student's $t$-test was used to compare the IOP between groups.

\section{Results}

\section{In vitro testing}

The results of the in vitro test are plotted in Figure 4 . With the membrane face intact, there was an average of $25.5 \pm 0.3 \mu \mathrm{l}$ BSS drained, with a mean flow rate of $0.9 \mu \mathrm{l} / \mathrm{s}$. As the membrane face was progressively opened with laser, the flow correspondingly increased in accordance with Poiseuille's law. With the membrane face completely open, the total BSS drained averaged $4023.3 \pm 38.4 \mu \mathrm{l}$ and a flow rate of $134.1 \mu \mathrm{l} / \mathrm{s}$. Moving from the closed position to the fully open position, there exists three orders of magnitude difference in the potential flow through the LL-GDD. Although this flow rate is much higher than would be needed clinically, it demonstrates the ability of the device to overcome resistance around the reservoir plate, which may develop years after implantation.

\section{In vivo testing}

During the 55 days following surgery, none of the study or control eyes showed signs of inflammation, infection, or cataract formation on ophthalmologic examination. At baseline, there was no difference in IOP between the control and surgical group (16.8 vs $16.7 \mathrm{~mm} \mathrm{Hg}, P=0.49$ ). Immediately after the surgery, the IOP in the LL-GGD surgical group dropped an average of $5.5 \mathrm{~mm} \mathrm{Hg}$, 


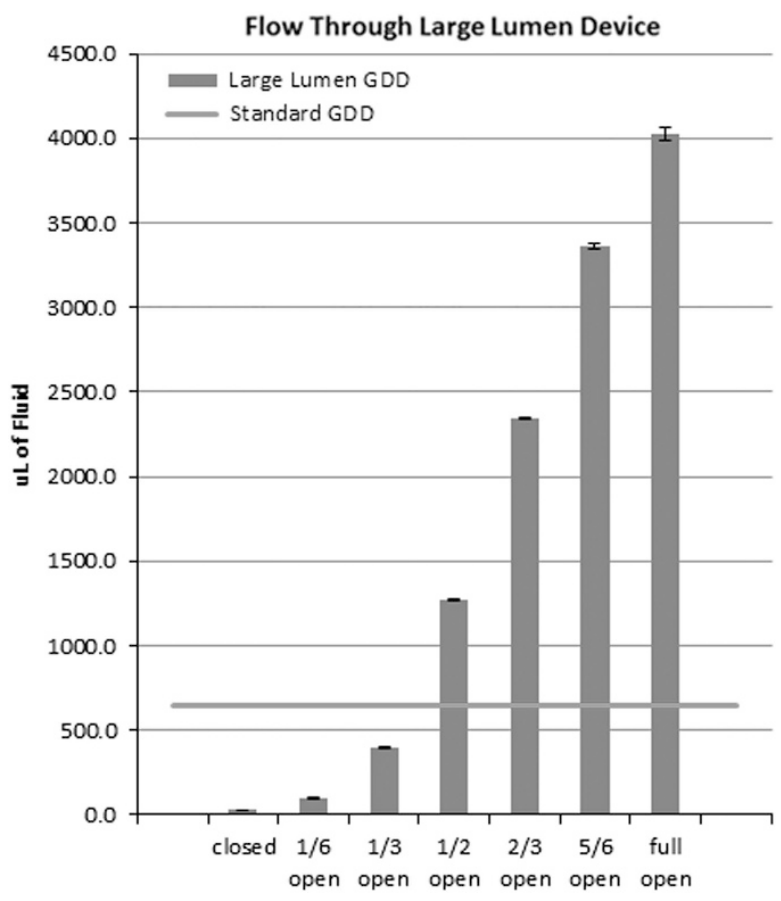

Figure 4 The flow through the LL-GDD increases exponentially as the membrane cap is opened with laser (blue bars). For comparison, the flow of a standard glaucoma drainage device is depicted by the red bar.

a statistically significant reduction $(P=0.001)$ that was maintained until the membrane laser procedure at week 5 . Despite having a tube with over four times the flow capacity of a conventional glaucoma drainage device, the IOP never dropped precipitously, and no choroidal effusions occurred. It is important to note that the membrane regulator face was completely intact during the first 5 weeks, indicating that the passive flow across the intact membrane was sufficient to have a significant effect on IOP.

At week 5, half of the membrane face was ruptured using argon laser. This resulted in an immediate increase in flow as evidenced by a fluid bleb over the reservoir plate, and a reduction in the IOP by an average of $1.8 \mathrm{~mm} \mathrm{Hg}$ in the surgical group. The 2 weeks following the initial 50\% membrane opening, the average IOP in the control group ranged from 4 to $9 \mathrm{~mm} \mathrm{Hg}$ lower than the control group.

At week 7 , the average IOP in the surgical group was $11 \mathrm{~mm} \mathrm{Hg}$ compared with $18 \mathrm{~mm} \mathrm{Hg}$ in the control group $(P<0.001)$. A second laser procedure was done to completely open the membrane face, which resulted in an immediate drop in the average IOP of the surgical group by another $2.7 \mathrm{~mm} \mathrm{Hg}$, which was maintained until the study termination at day 55 (Figure 5).

During the 8 weeks following surgery, none of the surgical or control eyes showed signs of inflammation, infection or cataract formation on ophthalmologic examination.

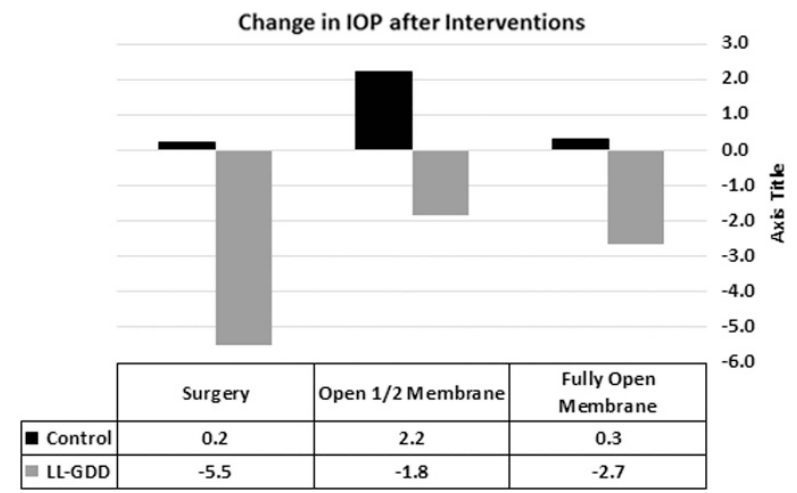

Figure 5 The IOP in the surgery group dropped after the initial surgery $(-5.5 \mathrm{~mm} \mathrm{Hg})$, the first membrane lasering $(-1.8 \mathrm{~mm} \mathrm{Hg})$ and the second membrane lasering $(-2.7 \mathrm{~mm} \mathrm{Hg})$ demonstrating an ability to lower the IOP non-invasively on-demand.

\section{Discussion}

The tube versus trabeculectomy study demonstrated a clear benefit to patients for tubes with fewer complications and a longer duration of effect. Still, these surgeries have a very high rate of failure at 5 years, usually related to underfiltration or decreased flow. This often necessitates either a revision of the patient's current tube, placement of a second tube, or photoablative surgery such as endoscopic cyclophotocoagulation to keep the IOP under control and prevent further vision loss. ${ }^{4,12}$

Glaucoma drainage devices provide surgeons a means to lower IOP in patients with medically uncontrolled glaucoma. However, their high rate of failure limits their long-term utility. The purpose of this study was to evaluate a next-generation glaucoma drainage device with quadrupling the flow capacity of standard GDDs, as well as the ability to adjust both the postoperative flow, and the placement of the tube tip in the anterior chamber.

The large lumen drainage device described here is designed to address the two major factors limiting the clinical utility of current GDDs: (1) preventing postoperative hypotony; (2) extending the device's functional duration. The first goal is accomplished with the flow restrictor membranes over the lumen of the LL-GDD. This restricts aqueous flow through the tube until the surgeon has determined that the eye is stable, and the membrane can then be opened non-invasively with laser or mechanically with a needle. The second goal is achieved by having a large lumen device, in effect-quadrupling the overall efficacy and potential drainage capability of the device. Whether 5 months or 5 years after the initial surgery, this additional flow can be tapped into as a means to further reduce the patient's IOP as dictated by clinical need.

It should also be noted that the membranes regulate flow when completely intact, but do not completely block it - which is a distinct design advantage. This means that 
there will be a continual, albeit low, flow of aqueous through the second unopened LL-GDD. This prevents blockage or failure of the tube, as well as minimizing the chance of infection.

In terms of controlling IOP, the LL-GDD has several distinct advantages. First, the membrane regulator prevents overfiltration and hypotony in the early postoperative period. Second, additional flow can be tapped into by physically opening the membrane face-we have demonstrated that this can be done either mechanically with a needle or non-invasively with laser.

In summary, the large-lumen glaucoma drainage device clearly demonstrated an ability both to prevent immediate postoperative hypotony and to allow progressively lower IOP in this proof-of-concept study. Eight weeks after the initial surgery, the animals exhibited no adverse effects, and the surgical group maintained a statistically significant lowering of IOP. Additional studies are underway to further characterize the surgical utility and biocompatibility of this nextgeneration aqueous flow device in the management of glaucoma.

\section{Summary}

What was known before

- The most common early complications of tube shunt implantation in glaucoma surgery is hypotony and associated problems.

What this study adds

- The large-lumen glaucoma drainage device demonstrated an ability both to prevent immediate postoperative hypotony and to allow progressively to lower the intraocular pressure.

\section{Conflict of interest}

The authors declare no conflict of interest.

\section{Acknowledgements}

Funding support was provided by the Colorado Bioscience Association. The study was conducted at the
University of Colorado facilities and was funded by the Colorado Bioscience Discovery Grant.

\section{References}

1 Oliver JE, Hattenhauer MG, Herman D, Hodge DO, Kennedy R, Fang-Yen $\mathrm{M}$ et al. Blindness and glaucoma: a comparison of patients progressing to blindness from glaucoma with patients maintaining vision. Am J Ophthalmol 2002; 133(6) 764-772.

2 Boland MV, Ervin AM, Friedman DS, Jampel HD, Hawkins BS, Vollenweider D et al. Comparative effectiveness of treatments for open-angle glaucoma: a systematic review for the U.S. Preventive Services Task Force. Ann Intern Med 2013; 158(4) 271-279.

3 Fremont AM, Lee PP, Mangione CM, Kapur K, Adams JL, Wickstrom SL et al. Patterns of care for open-angle glaucoma in managed care. Arch Ophthalmol 2003; 121(6) 777-783.

4 Gedde SJ, Schiffman JC, Feuer WJ, Herndon LW, Brandt JD, Budenz DL et al. Treatment outcomes in the Tube Versus Trabeculectomy (TVT) study after five years of follow-up. Am J Ophthalmol 2012; 153(5) 789-803. e2.

5 Eldaly MA, Bunce C, Elsheikha OZ, Wormald R. Non-penetrating filtration surgery versus trabeculectomy for open-angle glaucoma. Cochrane Database Syst Rev 2014; 2: Cd007059.

6 Samuelson TW, Katz LJ, Wells JM, Duh YJ, Giamporcaro JEUS iStent Study Group. Randomized evaluation of the trabecular micro-bypass stent with phacoemulsification in patients with glaucoma and cataract. Ophthalmology 2011; 118(3) 459-467.

7 Gedde SJ, Schiffman JC, Feuer WJ, Herndon LW, Brandt JD, Budenz DL et al. Three-year follow-up of the tube versus trabeculectomy study. Am J Ophthalmol 2009; 148(5) 670-684.

8 Gedde SJ, Herndon LW, Brandt JD, Budenz DL, Feuer WJ, Schiffman JC. Surgical complications in the Tube Versus Trabeculectomy Study during the first year of follow-up. Am J Ophthalmol 2007; 143(1) 23-31.

9 Budenz DL, Barton K, Gedde SJ, Feuer WJ, Schiffman J, Costa VP et al. Five-year treatment outcomes in the ahmed baerveldt comparison study. Ophthalmology 2015; 122(2) 308-316.

10 Olson JL, Velez-Montoya R, Bhandari R. Laser activated flow regulator for glaucoma drainage devices. Transl Vis Sci Technol 2014; 3(6) 3.

11 McEWEN WK. Application of Poiseuille's law to aqueous outflow. AMA Arch Ophthalmol 1958; 60(2) 290-294.

12 Bailey AK, Sarkisian SR Jr. Complications of tube implants and their management. Curr Opin Ophthalmol 2014; 25(2) 148-153. 